\title{
MARTINGALE INTEGRALS
}

\author{
BY \\ P. WARWICK MILLAR( $\left.{ }^{1}\right)$
}

1. Introduction. Let $(\Omega, F, P)$ be a probability space, and let $\{F(t), t \in T\}$, where $T$ is some (possibly infinite) interval on the real line, be an increasing family of sub-sigma-fields of $F$. Let $X=\{X(t), F(t), t \in T\}$ be a martingale, and $v$ $=\{v(t), t \in T\}$ a stochastic process where $v(t)$ is measurable $F(t)$ for each $t$. Stochastic integrals of the form $\int_{A} v(t) d X(t), A \subset T$, have long been studied under various hypotheses on $v$ and $X$. Early studies considered such integrals in the case where $X$ was Brownian motion, while a general theory of such integrals was begun by Doob in [4]. More recent contributions to the theory may be found, for example, in Meyer [6] and in Courrège [3]. Common to these, and to other published works on the topic, is the hypothesis that the martingale $X$ be square integrable. In the present paper, we remove the hypothesis of square integrability and develop a theory of stochastic integrals where the martingale may be only $L_{p}$ integrable, $1 \leqq p<\infty$. An independent study of stochastic integrals for nonsquare integrable martingales has been made by P. A. Meyer [8] using other methods.

The approach presented here depends on the concept of a martingale transform, discussed by Burkholder [2], and defined as follows. Let $\left\{F_{n}, n=0,1, \ldots\right\}$ be an increasing family of sub-sigma-fields of $F, f=\left\{f_{n}, F_{n}, n=1,2, \ldots\right\}$ a martingale, and $v=\left\{v_{n}, n=1,2, \ldots\right\}$ a stochastic process (called a multiplier sequence) with $v_{n}$ measurable $F_{n-1}$. Let $d_{1}=f_{1}, d_{2}=f_{2}-f_{1}, \ldots$ Then $g=\left\{g_{n}, n=1,2, \ldots\right\}$ is the transform of $f$ under $v$, provided that $g_{n}=\sum v_{k} d_{k}$. Suppose now we are given a continuous parameter martingale $X=\{X(t), 0 \leqq t<\infty\}$, and $v=\{v(t), 0 \leqq t<\infty\}$ a step function defined by

$$
\begin{aligned}
v(t, \omega) & =0, & & t<a_{1}, \\
\cdot & =v(j, \omega), & & a_{j} \leqq t<a_{j+1}, \\
& =0, & & a_{n} \leqq t,
\end{aligned}
$$

where $a_{1}<\cdots<a_{n}$, and $v(j)$ is measurable with respect to $F\left(a_{j}\right)$. Then the stochastic integral $\int v(t) d X(t)$ may be defined in this case to be

$$
\sum v(j)\left[X\left(a_{j+1}\right)-X\left(a_{j}\right)\right]
$$

Received by the editors April 6, 1967.

( ${ }^{1}$ These results are taken from the author's doctoral dissertation, written under the direction of D. L. Burkholder, and submitted to the University of Illinois. The research was supported by a terminal year National Science Foundation Graduate Fellowship.

Presently at the Department of Statistics, University of California, Berkeley. 
This expression, of course, closely resembles the martingale transform just defined. The basic idea of the present paper, then, is to regard the stochastic integral as, more or less, a species of martingale transform. This point of view makes available for us the martingale transform theory developed in [2].

In $\S 2$ we establish the existence of $\int v(t) d X(t)$ as a limit in $L_{p}$ norm, $1<p<\infty$, whenever the martingale $X$ satisfies $\sup _{t \in T} E|X(t)|^{p}<\infty$, and $v$ satisfies certain other hypotheses. In $\S 3$, an analogous result is established for $L_{1}$ martingales, the integral this time being a limit in probability, and, in general, not necessarily an element of $L_{1}$. Extension of this theory to interval parameter sets other than $[0, \infty)$ is routine.

$\$ 4$ discusses the processes $Y=\{Y(t), 0 \leqq t<\infty\}$ where $Y(t)=\int_{0}^{t} v(s) d X(s)$. Under certain hypotheses on $v$, it is shown that if $1<p<\infty$ and if the martingale $X$ satisfies $E|X(t)|^{p}<\infty$ for each $t$, then the process $Y$ is also a martingale with $E|Y(t)|^{p}<\infty$. It is worth pointing out that, although results of this nature have been known for $p=2$, they have not been established even for $p>2$. In case $X$ is an $L_{1}$ martingale, the $Y$ process satisfies an upcrossing inequality, and, in fact, $\lim _{t \rightarrow \infty} Y(t)$ exists a.s. and is finite. In $\S 5$ the results of $\$ 2,3$, and 4 are extended to include stochastic integrals defined by more general step functions than the ones previously introduced, and a much wider class of processes that may serve as integrands is thereby found. If $1<p<\infty$, and $X$ is an $L_{p}$ martingale, a norm on the step functions is found with the property that the step functions converge in the sense of this norm if and only if the corresponding stochastic integrals converge in $L_{p}$ norm.

$\S 6$ considers martingales $X=\{X(t), t \in[a, b]\}$ which have continuous paths. Let $\left(\pi_{m}\right)$, where $\pi_{m}=\left\{t_{m k}: a \leqq t_{m 1}<\cdots<t_{m s} \leqq b\right\}$ be a sequence of partitions of $[a, b]$ with $\lim _{m \rightarrow \infty} \max _{j}\left[t_{m, j+1}-t_{m, j}\right]=0$, and define $S_{m}(X)$, the quadratic variation of $X$ corresponding to $\pi_{m}$, by

$$
S_{m}^{2}(X)=\left[X\left(t_{m 1}\right)\right]^{2}+\left[X\left(t_{m 2}\right)-X\left(t_{m 1}\right)\right]^{2}+\cdots+\left[X\left(t_{m s}\right)-X\left(t_{m, s-1}\right)\right]^{2} .
$$

Then, if $1<p<\infty$, we show $\lim _{m \rightarrow \infty} S_{m}(X)=S(X)$ in $L_{p}$ norm, if $X$ is an $L_{p}$ martingale, and as a limit in probability if $p=1$. This generalizes part of a result known to be true if $X$ is Brownian motion. In fact, it is even true that there exist positive constants $M_{p}$ and $N_{p}$, depending on $p$ only, such that if $1<p<\infty$, then

$$
M_{p} E S(X)^{p} \leqq E X(b)^{p} \leqq N_{p} E S(X)^{p} .
$$

A result of Fisk [5] to the effect that, if $X$ has almost all paths continuous and of bounded variation, then $P\{X(t) \equiv X(0), t \in[a, b]\}=1$, follows from this inequality. $\$ 7$ develops a theory of quadratic variation for martingales having a.s. right continuous paths. This theory is applied in $\$ 8$ to give a martingale integral which in some ways improves that of $\$ 2,3$, and 5 .

Throughout this paper a stochastic process $X=\{X(t), t \in T\}$ will be called $L_{p}$ bounded if $\sup _{t} E|X(t)|^{p}<\infty$. If $X(t)$ is measurable $F(t)$ for each $t$, the process $X$ 
is said to be adapted to the $F(t)$ family. It will be assumed that $T$, the parameter set for the martingales discussed below, is the interval $[0, \infty)$, unless specified otherwise.

2. Stochastic integrals for $L_{p}, 1<p<\infty$. We define an extended $t-\omega$ step function $v=\{v(t), t \in[0, \infty)\}$ as follows. If $0 \leqq a_{0}<a_{1}<\cdots$, (where $a_{n}$ may approach $\infty)$, then

$$
v(t)=v(j) \text { for } a_{j} \leqq t<a_{j+1}
$$

where $v(j)$ is measurable $F\left(a_{j}\right)$. The integral of the step function $v$ with respect to the martingale $X=\{X(t), F(t), 0 \leqq t<\infty\}$ is

$$
\sum v(j)\left[X\left(a_{j+1}\right)-X\left(a_{j}\right)\right]
$$

which will be denoted by $\int v(t) d X(t)$. If $X$ is $L_{1}$ bounded, and $\sup _{t \in T}|v(t)|<\infty$, then the expression (1) is well defined almost surely by Theorem 1 of Burkholder [2]. If $X$ is $L_{p}$ bounded for some $p, 1<p<\infty$, and if $v$ is uniformly bounded, then it is well defined as both an a.s. and $L_{p}$ limit by [2, Theorem 9].

If $z=\{z(t), t \in T\}$ is a real, separable process, we define the $e-s$ norm of $z$ by

$$
\|z\|_{e-s}=E^{(1 / 4)} \sup |z(t, \omega)|^{4} \text {. }
$$

An important special case is $z(t, \omega)=z(t)$, where the $e-s$ norm reduces to the supremum norm.

The space of bounded, extended $t-\omega$ step functions is a vector space. We shall be interested in the completion of this vector space under the $e-s$ norm. In this vein, we state without proof the following simple proposition.

Proposition 2.1. If $v=\{v(t), t \in[a, b]\}$ is a uniformly bounded stochastic process adapted to the $F(t)$ family and having a.s. continuous paths, then $v$ is in the $e-s$ completion of the $t-\omega$ step functions.

All theorems in $\$ \$ 2$ through 4 will be proved for processes $v$ in the $e-s$ completion of the space of $t-\omega$ step functions. In $\$ 5$, more general step functions will be introduced with the purpose of extending the class of processes $v$ which can serve as integrands in the stochastic integrals.

LEMMA 2.2. Let $X=\{X(t), F(t), t \in T\}$ be an $L_{4}$ bounded martingale. Let $v_{n}=\left\{v_{n}(t), t \in T\right\}$ be a sequence of $t-\omega$ step functions converging in $e-s$ norm. Then the sequence of integrals $\int v_{n}(t) d X(t)$ converges in $L_{2}$ norm.

Proof. Let the step functions $v_{n}$ have the representation

$$
v_{n}(t)=v_{n}(j) \text { for } a_{n j} \leqq t<a_{n, j+1},
$$

and let $\theta_{n}$ be the integral of $v_{n}$ with respect to $X$. Then

$$
E\left[\theta_{m}-\theta_{n}\right]^{2}=E\left[\sum\left\{v_{n}(j)-v_{m}(j)\right\}\left\{X\left(a_{j+1}\right)-X\left(a_{j}\right)\right\}\right]^{2}
$$


where the partitions of the line corresponding to $v_{n}$ and to $v_{m}$ have been intermeshed and relabeled. This is equal to

$$
\begin{aligned}
E\left[\sum \left\{v_{n}(j)-\right.\right. & \left.\left.v_{m}(j)\right\}^{2}\left\{X\left(a_{j+1}\right)-X\left(a_{j}\right)\right\}^{2}\right] \\
& \leqq E\left[\sup _{j}\left|v_{n}(j)-v_{m}(j)\right|^{2} \sum\left\{X\left(a_{j+1}\right)-X\left(a_{j}\right)\right\}^{2}\right] \\
& \leqq E^{(1 / 2)} \sup \left|v_{n}(j)-v_{m}(j)\right|^{4} E^{(1 / 2)}\left[\sum\left\{X\left(a_{j+1}\right)-X\left(a_{j}\right)\right\}^{2}\right]^{2} \\
& \leqq N_{4}\left\|v_{n}-v_{m}\right\|_{e-s}^{2} E^{(1 / 2)}|X(\infty)|^{4}
\end{aligned}
$$

where the last inequality follows from Burkholder's Theorem 9 [2], with $p$ equal to 4 , and where $X(\infty)$ is equal to $\lim _{t \rightarrow \infty} X(t)$.

REMARK. While Lemma 2.2 may appear weaker in certain respects than the known results for $L_{2}$ martingales, it nevertheless has the virtue that the properties of the step functions $v_{n}$ are formulated independently of the martingale. This will be important for the following theorems.

If, in Lemma 2.2, $v_{n} \rightarrow v$ in $e-s$ norm, we will denote the limit of the sequence of integrals $\int v_{n}(t) d X(t)$ by $\int v(t) d X(t)$. This random variable, as a limit in $L_{2}$ norm, is defined uniquely (except for an $\omega$-set of probability zero) and is independent of the sequence of $v_{n}$ chosen, since two sequences converging to $v$ in $e-s$ norm can be combined to form a single sequence which converges to $v$, with the corresponding sequence of step function integrals converging in $L_{2}$ norm. This lemma thus allows the definition of stochastic integral to be extended to all processes $v$ of finite $e-s$ norm, which are limits in $e-s$ norm of $t-\omega$ step functions. By Proposition 2.1, the uniformly bounded, adapted, a.s. continuous processes belong to this class whenever the martingale is restricted to a compact interval.

THEOREM 2.3. Let $1<p<\infty$. Let $X=\{X(t), F(t), t \in T\}$ be an $L_{p}$ bounded martingale, and let $v=\{v(t), t \in T\}$ be in the $e-s$ closure of the $t-\omega$ step functions with $|v(t, \omega)| \leqq 1$. Then $\int v(t) d X(t)$ exists in the sense of an $L_{p}$ limit. More precisely, if $v_{n}$ is any sequence of $t-\omega$ step functions converging to $v$ in $e-s$ norm, then the integrals $\int v_{n}(t) d X(t)$ converge in $L_{p}$ norm to a (unique) limit, denoted by $\int v(t) d X(t)$.

Remark. The condition $|v(t, \omega)| \leqq 1$ can be weakened, as Theorem 2.4 shows; the price paid for this is that convergence of $\int v_{n}(t) d X(t)$ takes place in a weaker sense.

Proof. We recall the following fact, due to Burkholder [2, proof of Theorem 9]: there is a constant $N_{p}$ depending on $p$ only, $1<p<\infty$, such that if $f=\left(f_{1}, f_{2}, \ldots\right)$ is a martingale, and $v=\left(v_{1}, v_{2}, \ldots\right)$ is any multiplier sequence with $\sup _{n}\left|v_{n}\right| \leqq 1$, then

$$
E\left|g_{n}\right|^{p} \leqq N_{p} E\left|f_{n}\right|^{p}
$$

where $g=\left(g_{1}, g_{2}, \ldots\right)$ is the transform of $f$ under $v$.

Assume first that $1<p \leqq 2$. Since $X$ is $L_{p}$ bounded for some $p, 1<p<\infty$, it follows that $X$ has a last element $X(\infty)$, and in fact, $X(t)=E\{X(\infty) \mid F(t)\}$. For each 
integer $n$, let $I_{n}$ be the indicator of the interval $[-n, n]$, and set $X^{n}(\infty)=X(\infty) I_{n}$ $\circ X(\infty)$, where " $\circ$ " denotes composition of functions. Define, for each $n$, a uniformly bounded martingale $X^{n}=\left\{X^{n}(t), t \in T\right\}$ by $X^{n}(t)=E\left\{X^{n}(\infty) \mid F(t)\right\}$. Let $v_{m}$ be a sequence of step functions, $\left|v_{m}(t, \omega)\right| \leqq 1$, converging to $v$ in $e-s$ norm; let $\left\{t_{m k}\right\}$, such that $0 \leqq t_{m 1}<t_{m 2}<\cdots$, be the partition of $[0, \infty)$ corresponding to $v_{m}$. Let $\theta_{m}^{n}$ be the integral of $v_{m}$ with respect to $X^{n}$ :

$$
\theta_{m}^{n}=\sum_{k} v_{m}\left(t_{m k}\right)\left[X^{n}\left(t_{m, k+1}\right)-X^{n}\left(t_{m k}\right)\right] .
$$

Since $X^{n}$ is uniformly bounded, it follows from Lemma 2.2 that

$$
\lim _{m \rightarrow \infty} \theta_{m}^{n}=\int v(t) d X^{n}(t)=\theta^{n}
$$

in $L_{2}$ norm, for each $n$. Here we have used the fact that the $e-s$ norm convergence of the $v_{n}$ does not depend on the particular martingale being transformed.Moreover, since $1<p \leqq 2$, the convergence takes place in $L_{p}$ norm as well. However,

$$
\lim _{n \rightarrow \infty} \theta_{m}^{n}=\sum_{k} v_{m}\left(t_{m k}\right)\left[X\left(t_{m, k+1}\right)-X\left(t_{m k}\right)\right]
$$

exists in the sense of $L_{p}$ convergence, and in fact, the convergence is uniform in $m$. For

$$
\begin{aligned}
E\left|\theta_{m}^{l}-\theta_{m}^{j}\right|^{p} & =E\left|\sum v_{m}\left(t_{m k}\right)\left[X^{l}\left(t_{m, k+1}\right)-X^{l}\left(t_{m k}\right)\right]-\sum v_{m}\left(t_{m k}\right)\left[X^{j}\left(t_{m, k+1}\right)-X^{j}\left(t_{m k}\right)\right]\right|^{p} \\
& =E \mid \sum v_{m}\left(t_{m k}\right)\left[E\left(\left\{X^{l}(\infty)-X^{j}(\infty)\right\} \mid F\left(t_{m, k+1}\right)\right)\right. \\
& \left.-E\left(\left\{X^{l}(\infty)-X^{j}(\infty)\right\} \mid F\left(t_{m k}\right)\right)\right]\left.\right|^{p} .
\end{aligned}
$$

The expression under the summation sign is just the transform, under $v_{m}\left(t_{m k}\right)$, of the martingale $Y=\left\{Y_{k}, k=1,2, \ldots\right\}$, where

$$
Y_{k}=E\left\{\left[X^{l}(\infty)-X^{j}(\infty)\right] \mid F\left(t_{m k}\right)\right\} .
$$

Hence, by (2),

$$
\begin{aligned}
E\left|\theta_{m}^{l}-\theta_{m}^{j}\right|^{p} & \leqq N_{p} E|Y(\infty)|^{p} \\
& \leqq N_{p} E\left|X^{l}(\infty)-X^{j}(\infty)\right|^{p} \\
& \left.\leqq N_{p} \int_{(|X(\infty)|>\})}|X(\infty)|^{p}, \quad \text { (assuming } l>j\right)
\end{aligned}
$$

which is independent of $m$, and approaches zero as $j$ and $l$ increase, since $X(\infty)$ is $L_{p}$ integrable.

It follows that, if $v_{n}$ is a sequence of $t-\omega$ step functions bounded by 1 and converging in $e-s$ norm, then the sequence of corresponding integrals with respect to $X$ converges in $L_{p}$ norm. For if $\theta_{r}$ is the integral of $v_{r}$ with respect to $X$, we have

$$
\left\|\theta_{r}-\theta_{s}\right\|_{p} \leqq\left\|\theta_{r}-\theta_{r}^{n}\right\|_{p}+\left\|\theta_{r}^{n}-\theta_{s}^{n}\right\|_{p}+\left\|\theta_{s}^{n}-\theta_{s}\right\|_{p}
$$


where $\|f\|_{p}=\left[E|f|^{p}\right]^{(1 / p)}$. Pick $n$ so large that the first and third terms on the right are each less than $\varepsilon / 3$. This can be done since (5a), (5b) imply that $\lim _{n \rightarrow \infty}[p] \theta_{r}^{n}=\theta_{r}$ occurs uniformly in $r$. With $n$ so chosen, pick $r, s$ so large that the second term is less than $\varepsilon / 3$, justifying this choice with the argument following equation (4). This establishes the theorem for the case $1<p \leqq 2$.

Suppose next that $X$ is an $L_{p}$ bounded martingale, $2<p<\infty$. If, for each $n, X^{n}$ is defined as before, and if $\theta_{m}^{n}$ is defined by (4), then $X^{n}$ is a uniformly bounded martingale, and $\lim _{m \rightarrow \infty} \theta_{m}^{n}=\theta^{n}$ exists as an $L_{2}$ limit, and thus as a limit in probability. If $p^{\prime}>p$, then it follows from (2) that $E\left|\theta_{m}^{n}\right|^{p^{\prime}} \leqq N_{p^{\prime}} E\left|X^{n}(\infty)\right|^{p^{\prime}}$, whence for each $n$, the sequence $\left|\theta_{m}^{n}\right|^{p}$ is uniformly integrable. Hence, for each $n, \lim _{m \rightarrow \infty} \theta_{m}^{n}=\theta^{n}$ occurs in the sense of convergence in $L_{p}$ norm. We now have statement (4) for $2<p<\infty$; the rest of the proof now proceeds as in the case $1<p \leqq 2$ already discussed.

Next we will examine conditions under which the stochastic integral with respect to an $L_{p}$ martingale, $1<p<\infty$, may be asserted to exist as a limit in $L_{1}$ norm, when the condition $|v(t)| \leqq 1$ is not assumed. To do this we will need the following lemma.

Lemma 2.4. Let $1<p<\infty$, and let $f=\left(f_{1}, f_{2}, \ldots\right)$ be an $L_{p}$ bounded martingale. Let $v=\left(v_{1}, v_{2}, \ldots\right)$ be a multiplier sequence satisfying

$$
E\left(v^{*}\right)^{q+\delta}<\infty,
$$

where $v^{*}=\sup _{n}\left|v_{n}\right|,(1 / p)+(1 / q)=1$, and $\delta$ is some positive number. If $g=\left(g_{1}, g_{2}, \ldots\right)$ is the transform of $f$ under $v$, then there exist constants $p^{\prime}, r, R$, depending on $p$ and $\delta$ only, with $p^{\prime}>1$, such that

$$
\left\|g_{n}\right\|_{1} \leqq\left\|g_{n}\right\|_{p^{\prime}} \leqq R\left\|v^{*}\right\|_{a+\delta}^{r}\left\|f_{n}\right\|_{p^{\prime}}
$$

Proof. Let $p^{\prime}=\left[p^{2}(1+\delta)-\delta p\right] /\left[p^{2}+\delta p-\delta\right]$. It is easily checked that if $p>1$, then $p^{\prime}>1$; and that we can assume $\delta$ so small that $p^{\prime}<p$. By Theorem 9 of Burkholder [2]

$$
\begin{aligned}
E\left|g_{n}\right|^{p^{\prime}} & \leqq N_{p^{\prime}} E S_{n}(g)^{p^{\prime}} \leqq N_{p^{\prime}} E\left[\left(v^{*}\right)^{p^{\prime}} S_{n}(f)^{p^{\prime}}\right] \\
& \leqq N_{p^{\prime}}\left[E\left(v^{*}\right)^{\left.p^{\prime} q^{*}\right]^{\left(1 / q q^{\prime}\right)}}\left[E S_{n}(f)^{p^{\prime} p^{*}}\right]^{\left(1 / p^{\prime \prime}\right)}\right.
\end{aligned}
$$

where $\left(1 / p^{\prime \prime}\right)+\left(1 / q^{\prime \prime}\right)=1$. Set $p^{\prime \prime}=p / p^{\prime}$, so that $q^{\prime \prime}=p^{\prime \prime} /\left(p^{\prime \prime}-1\right)=p /\left(p-p^{\prime}\right)$, which implies that $p^{\prime} q^{\prime \prime}=p^{\prime} p /\left(p-p^{\prime}\right)=p /(p-1)+\delta$. This yields

$$
\begin{aligned}
E\left|g_{n}\right|^{p^{\prime}} & \leqq N_{p^{\prime}}\left[E\left(v^{*}\right)^{q+\delta}\right]^{\left(p-p^{\prime}\right) / p}\left[E S_{n}(f)^{p}\right]^{p^{\prime} / p} \\
& \leqq\left[\left(N_{p^{\prime}} \mid M_{p}\right)^{p / p^{\prime}}\right]\left[E\left(v^{*}\right)^{q+\delta}\right]^{p-p^{\prime} / p}\left[E\left|f_{n}\right|^{p}\right]^{p^{\prime} / p},
\end{aligned}
$$

which implies the result.

The lemma obviously implies that the transform of an $L_{p}(1<p<\infty)$ bounded martingale under a multiplier sequence satisfying the given condition is uniformly integrable. 
THEOREM 2.5. Let $1<p<\infty$. Let $X=\{X(t), t \in T\}$ be an $L_{p}$ bounded martingale, and let $v$ be a process in the closure of the step functions under the norm $E^{(1 / 2 q)} \sup _{t}|v(t, \omega)|^{2 q}$, where $(1 / p)+(1 / q)=1$. Then $\int v(t) d X(t)$ exists as an $L_{1}$ limit.

Proof. The proof follows the method of Theorems 2.2 and 2.3, and will therefore only be sketched. First, with notation as in Theorem 2.2, one proves that

$$
E\left[\theta_{m}-\theta_{n}\right]^{2} \leqq N_{2 p} E^{(1 / q)} \sup _{j}\left|v_{n}(j)-v_{m}(j)\right|^{2 q} E^{(1 / p)}|X(\infty)|^{2 p},
$$

which establishes the result for $L_{2 p}$ bounded martingales (and, in particular, uniformly bounded ones). One then replaces statement (2) of the proof of Theorem 2.3 by statement (6) of Theorem 2.4, with $\delta=q$, and proceeds as before.

REMARK. For a different method of extending the class of $v$ which can be integrated, the reader is referred to $\S 5$, and to $\S 8$.

REMARK. The integral developed in this section (and also the integrals of $\$ \$ 3$ and 5) will not necessarily be countably additive. For an integral that does better in this respect, the reader is referred to $\S 8$.

3. Stochastic integrals for $L_{1}$. In this section we consider under what conditions and in what sense stochastic integrals may be developed for martingales $X$ which are $L_{1}$ bounded. Attempts to obtain them as $L_{1}$ limits will be frustrated by the fact that Theorem 9 of [2] does not hold in the present situation. In fact, there exist a martingale $X$ and a uniformly bounded sequence of $t-\omega$ step functions $v_{n}$ which converge to $v$ in $e-s$ norm, with the property that $\int v(t) d X(t)$ exists as a limit (a.s.) but the limit is not integrable (see [2, p. 1495]). This is true even if $X$ is assumed uniformly integrable. Thus, for the general $L_{1}$ case, we must seek another type of convergence instead of $L_{1}$ convergence. We develop here a theory of stochastic integrals wherein the integral is defined as a limit in probability.

Theorem 3.1. Let $X=\{X(t), t \in T\}$ be a martingale with a last element $X(\infty)$, so that $X(t)=E[X(\infty) \mid F(t)]$. Let $v_{n}$ be a sequence of step functions converging to $v$ in $e-s$ norm, and satisfying $\left|v_{n}(t, \omega)\right| \leqq 1$. Then the sequence $\int v_{n}(t) d X(t)$ converges in probability to a (unique) limit denoted by $\int v(t) d X(t)$.

Remark. Choose $s$ satisfying $0<s<\infty$. Let $X$ be any martingale with parameter set $[0, \infty)$, and let $v_{n}$ and $v$ satisfy the hypotheses of Theorem 3.1. Then $\{X(t), t \in[0, s)\}$ is a martingale having a last element $X\left(s^{-}\right)$. If $I(s)$ is the indicator of $[0, s)$, then $I(s) v_{n}(t)$ is a step function which converges to $I(s) v(t)$ in $e-s$ norm. The proof of Theorem 3.1 implies that $\int I(s) v_{n}(t) d X(t)$, which will be denoted by $\int_{0}^{s} v_{n}(t) d X(t)$, converges in probability to $\int I(s) v(t) d X(t)$. This establishes for each $s$ the existence of $\int_{0}^{s} v(t) d X(t)$. If $1<p<\infty$, and if $X$ is a martingale in $L_{p}$, then by Theorem $2.3 \int_{0}^{s} v(t) d X(t)$ exists as a limit in $L_{p}$ norm. Although, in general, $\int_{0}^{s} v(t) d X(t)$ may not be integrable, nevertheless it is proved in $\$ 4$ that if $X$ is $L_{1}$ bounded, then $\lim _{s \rightarrow \infty} \int_{0}^{s} v(t) d X(t)$ exists, and we denote this limit by $\int_{0}^{\infty} v(t) d X(t)$. 
Proof. As in Theorem 2.3, define for each $n$ the uniformly bounded martingale $X^{n}=\left\{X^{n}(t), t \in T\right\} \quad$ with $X^{n}(t)=E\left\{X(\infty) I_{n} \circ X(\infty) \mid F(t)\right\}=E\left\{X^{n}(\infty) \mid F(t)\right\}$, and let $\theta_{m}^{n}=\sum v_{m}\left(t_{m k}\right)\left[X^{n}\left(t_{m, k+1}\right)-X^{n}\left(t_{m k}\right)\right]$. Since $X^{n}$ is uniformly bounded, it follows from Lemma 2.2 that

$$
\lim _{m \rightarrow \infty} \theta_{m}^{n}=\int v(t) d X^{n}(t)=\theta^{n}
$$

exists as a limit in probability. Furthermore, for fixed $m$,

$$
\lim _{n \rightarrow \infty} \theta_{m}^{n}=\sum v_{m}\left(t_{m k}\right)\left[X\left(t_{m, k+1}\right)-X\left(t_{m k}\right)\right]=\theta_{m}
$$

exists as a limit in probability, and the convergence is uniform in $m$. To see this, we calculate

$$
\begin{aligned}
& P\left\{\left|\theta_{m}^{l}-\theta_{m}^{j}\right|>\lambda\right\}=P\left\{\mid \sum v_{m}\left(t_{m k}\right)\left[X^{i}\left(t_{m, k+1}\right)-X^{l}\left(t_{m k}\right)\right]\right.\left.-\sum v_{m}\left(t_{m k}\right)\left[X^{j}\left(t_{m, k+1}\right)-X^{j}\left(t_{m k}\right)\right] \mid>\lambda\right\} \\
&=P\left\{\mid \sum v_{m}\left(t_{m k}\right)\left[E\left\{X^{l}(\infty)-X^{j}(\infty) \mid F\left(t_{m, k+1}\right)\right\}\right.\right. \\
&\left.\left.-E\left\{X^{\prime}(\infty)-X^{j}(\infty) \mid F\left(t_{m k}\right)\right\}\right] \mid>\lambda\right\} .
\end{aligned}
$$

The expression under the summation sign is just the transform under $v_{m}\left(t_{m k}\right)$ of the martingale $Y=\left\{Y_{k}, k=1,2,3, \ldots\right\}$, where

$$
Y_{k}=E\left\{X^{l}(\infty)-X^{j}(\infty) \mid F\left(t_{m k}\right)\right\} .
$$

Hence, by [2, Theorem 6],

$$
\begin{aligned}
\lambda P\left\{\left|\theta_{m}^{l}-\theta_{m}^{j}\right|>\lambda\right\} & \leqq M E|Y(\infty)| \\
& \leqq M E\left|X^{l}(\infty)-X^{j}(\infty)\right| \\
& \leqq M \int_{(|X(\infty)|>j)}|X(\infty)| \quad \text { assuming } l>j .
\end{aligned}
$$

For fixed $\lambda$, the latter approaches zero as $j, l$ increase, independently of $m$. Therefore, if $\theta_{r}$ is the integral of $v_{r}$ with respect to $X$, we have

$$
P\left\{\left|\theta_{r}-\theta_{s}\right|>\lambda\right\} \leqq P\left\{\left|\theta_{r}-\theta_{r}^{n}\right|>\lambda / 3\right\}+P\left\{\left|\theta_{r}^{n}-\theta_{s}^{n}\right|>\lambda / 3\right\}+P\left\{\left|\theta_{s}^{n}-\theta_{s}\right|>\lambda / 3\right\} .
$$

Pick $n$ so large that the first and third terms are each less than $\varepsilon / 3$; this can be done independently of $r$ and $s$. After this is done, pick $r$ and $s$ so large that the remaining term is less than $\varepsilon / 3$. Since $\lambda$ is arbitrary, $\theta_{r}$ is Cauchy in probability, completing the proof.

If one imposes slightly stronger conditions on the martingale $X$, then one can actually obtain the existence of $\int v(t) d X(t)$ as an $L_{1}$ limit. To see this, we prove the following lemma.

LEMMA 3.2. Let $f=\left(f_{1}, f_{2}, \ldots\right)$ be a martingale which is $L \log ^{2} L$ bounded; (i.e. $\left.\sup _{n} E\left|f_{n}\right|\left[\log ^{+}\left|f_{n}\right|\right]^{2}<\infty\right)$ and let $v=\left(v_{1}, v_{2}, \ldots\right)$ be a multiplier sequence 
satisfying $\sup _{n}\left|v_{n}(\omega)\right| \leqq 1$. If $g=\left(g_{1}, g_{2}, \ldots\right)$ is the transform of $f$ under $v$, then the sequence $\left\{g_{n}\right\}$ is uniformly integrable (and converges in $L_{1}$ norm).

Proof. Referring to [9, Theorem 4.32, Vol. II, p. 118], let $\chi(u)=0$ for $u \leqq 1$, and $\chi(u)=u \log u$ for $u>1$. Then

$$
\phi(u)=u \int_{1}^{u}[(\log t) / t] d t=(1 / 2) u \log ^{2} u .
$$

Therefore, by the theorem just cited, if $T$ is a quasi-linear operator,

$$
\int|T f| \log ^{+}|T f| \leqq K \int|f|\left[\log ^{+}|f|\right]^{2}+K .
$$

Using the method of proof of [2, Theorems 9 and 10], we have

$$
\int\left|g_{n}\right| \log ^{+}\left|g_{n}\right| \leqq K \int\left|f_{n}\right|\left[\log ^{+}\left|f_{n}\right|\right]^{2}+K,
$$

which implies the lemma.

Actually slightly stronger results are possible. For example, if one takes $\chi(u)=0$ if $u \leqq e^{e}$, and $\chi(u)=u \log \log u$, if $u>e^{e}$, then one can obtain an analogous result assuming only that $f$ is $L \log L \log \log L$ bounded.

THEOREM 3.3. Let $X$ be an $L \log ^{2} L$ bounded martingale, and let $v$ be in the $e-s$ closure of the step functions, satisfying $|v(t)| \leqq 1$. Then $\int v(t) d X(t)$ exists as an $L_{1}$ limit.

Proof. If $v_{n}$ is a sequence of step functions converging to $v$ in $e-s$ norm, with $\left|v_{n}(t)\right| \leqq 1$, then $\int v_{n}(t) d X(t)$ converges to $\int v(t) d X(t)$ in probability, by Theorem 3.1. Since the sequence $\int v_{n}(t) d X(t)$ is uniformly integrable, by Theorem 3.2 , the theorem is immediate.

We close this section by giving an alternate proof of Theorem 3.1 which may be of some interest. Here we impose the additional hypotheses that $X$ have right continuous paths, and that the family $\{F(t)\}$ be right continuous. The proof rests on the Krickeberg decomposition of martingales, and on the Meyer decomposition of continuous parameter supermartingales [6]. Assuming that $X$ is an $L_{1}$ bounded martingale with right continuous paths, we prove $\int v(t) d X(t)$ exists as a limit in probability. Since $X$ is $L_{1}$ bounded, we can write $X=X^{\prime}-X^{\prime \prime}$, where $X^{\prime}, X^{\prime \prime}$ are each nonnegative martingales (the Krickeberg decomposition). Therefore, in proving the theorem, it suffices to consider only nonnegative martingales, since if this case is proved we can obtain the general case by linearity. With these assumptions, define $X^{n}(t)$ by $X^{n}(t)=\min [X(t), n]$. Then $X^{n}=\left\{X^{n}(t), t \in T\right\}$ is a uniformly bounded, right continuous supermartingale and, as such, has the decomposition

$$
X^{n}(t)=M^{n}(t)+A^{n}(t)
$$

where $M^{n}(t)$ is an $L_{2}$ bounded martingale, and $A^{n}(t)$ is an $L_{2}$ bounded increasing process (consult Meyer [7, Chapter VII]). Let $v_{m}$ be as in Theorem 3.1, and let 
$\theta_{m}^{n}, \beta_{m}^{n}, \alpha_{m}^{n}$, denote respectively the integrals of $v_{m}$ with respect to $X^{n}, M^{n}$, and $A^{n}$. Then $\theta_{m}^{n}=\beta_{m}^{n}+\alpha_{m}^{n}$. From Theorem 2.3, $\lim _{m \rightarrow \infty} \beta_{m}^{n}=\beta^{n}$ in $L_{2}$ norm; and since $A^{n}(t)$ is $L_{2}$ bounded and increasing, $\lim _{m \rightarrow \infty} \alpha_{m}^{n}=\alpha^{n}$ in $L_{1}$ norm whenever $v_{m} \rightarrow v$ in $e-s$ norm, $\left|v_{m}\right| \leqq 1$. Hence, $\theta_{m}^{n} \rightarrow \theta^{n}$ in $L_{1}$ norm. Also, by the definition of $X^{n}$, $\theta_{m}^{j}=\theta_{m}^{k}$ on the set $\left\{\sup _{t}|X(t)|<j\right\}$, assuming $k>j$. Therefore, $\lim _{k \rightarrow \infty} \theta_{m}^{k}=\theta_{m}$ in probability, and, in fact, the convergence is uniform in $m$, since

Also,

$$
P\left\{\left|\theta_{m}-\theta_{m}^{k}\right|>\varepsilon\right\} \leqq P\left\{\sup _{t}|X(t)|>k\right\} \leqq \sup _{t} E|X(t)| / k .
$$

$$
P\left\{\left|\theta_{m}-\theta_{n}\right|>\varepsilon\right\} \leqq P\left\{\left|\theta_{m}-\theta_{m}^{k}\right|>\varepsilon / 3\right\}+P\left\{\left|\theta_{m}^{k}-\theta_{n}^{k}\right|>\varepsilon / 3\right\}+P\left\{\left|\theta_{n}^{k}-\theta_{n}\right|>\varepsilon / 3\right\} .
$$

One then chooses $k$ so large that the first and third terms are each arbitrarily small; and, with $k$ so chosen, one then finds $m$ and $n$ so large that the middle term is arbitrarily small. Thus $\left\{\theta_{m}\right\}$ is Cauchy in probability, which establishes the result.

4. Martingale transforms. The preceding sections establish, among other things, the existence of $\int_{0}^{t} v(s) d X(s)$ for every $t, 0 \leqq t<\infty$, under various hypotheses on $X$ and $v$. The process $Y=\{Y(t), 0 \leqq t<\infty\}$ with $Y(t)=\int_{0}^{t} v(s) d X(s)$ will be called a martingale transform; in case the martingale $X$ is a discrete parameter martingale, this definition coincides with that of [2]. In this section we examine some properties of these processes.

TheOREM 4.1. Let $1<p<\infty$. Let $X=\{X(t), 0 \leqq t<\infty\}$ be a martingale with $E|X(t)|^{p}<\infty$ for every $t$. Let $v=\{v(t), 0 \leqq t<\infty\}$ be a process in the $e-s$ closure of the step functions, with $|v(t, \omega)| \leqq 1$. Then

(a) $Y$ is a martingale in $L_{p}$,

(b) $E|Y(t)|^{p} \leqq M_{p} E|X(t)|^{p}, M_{p}$ depending only on $p$,

(c) $Y$ is $L_{p}$ bounded if $X$ is.

Proof. (a) Let $v_{n}=\left\{v_{n}(t), 0 \leqq t<\infty\right\}$ be a sequence of step functions bounded by 1 and converging to $v$ in $e-s$ norm. Then $\lim _{n \rightarrow \infty} Y^{n}(s)=Y(s)$ in $L_{p}$ norm, where $Y^{n}(s)=\int_{0}^{s} v_{n}(t) d X(t)$. If $\Lambda \in F(s)$, and $s<s_{1}$, then $\int_{\Lambda} Y^{n}(s) d P=\int_{\Lambda} Y\left(s_{1}\right) d P$. Let $n \rightarrow \infty$ to obtain $\int_{\Lambda} Y(s) d P=\int_{\Lambda} Y\left(s_{1}\right) d P$, which implies $Y$ is a martingale. The bound in (b) is true if $v$ is a step function, by [2, Theorem 9], and so is true for $Y$ by the $L_{p}$ convergence of $Y^{n}(t)$ to $Y(t)$. Finally, (c) follows from (b).

In case $X$ is an $L_{1}$ martingale, the process $Y$.may not be integrable, as discussed in the beginning of \$3. However, we do have the following theorems, wherein we assume throughout, as we may, that we are dealing with a separable modification of the $Y$ process.

TheOREM 4.2. Let $X=\{X(t), 0 \leqq t<\infty\}$ be a martingale, and $v=\{v(t), 0 \leqq t<\infty\}$ a process in the $e-s$ completion of the step functions, satisfying $|v(t, \omega)| \leqq 1$. Then

$$
\lambda P\left\{\sup _{t}|Y(t)|>\lambda\right\} \leqq M \sup _{t} E|X(t)|
$$

where $M$ is independent of the martingale $X$ and the process $v$. 
Proof. In view of the assumed separability of $Y$, it suffices, by well-known arguments, to prove that for any finite sequence $t_{1}<t_{2}<\cdots<t_{N}$, we have

$$
\lambda P\left\{\sup _{1 \leqq k \leqq N}\left|Y\left(t_{k}\right)\right|>\lambda\right\} \leqq M \sup _{t} E|X(t)| .
$$

Let $v_{n}=\left\{v_{n}(t), 0 \leqq t<\infty\right\}$ be a sequence of step functions converging to $v$ in $e-s$ norm, and let $Y^{n}(t)=\int_{0}^{t} v_{n}(s) d X(s)$. Then, to prove (A) it suffices to prove

$$
\lambda P\left\{\sup _{1 \leqq k \leqq N}\left|Y^{n}\left(t_{k}\right)\right|>\lambda\right\} \leqq M \sup _{t} E|X(t)|
$$

for each $n$, because $\sup _{1 \leqq k \leqq N}\left|Y^{n}\left(t_{k}\right)\right| \rightarrow \sup _{1 \leqq k \leqq N}\left|Y\left(t_{k}\right)\right|$ in probability, by virtue of Theorem 3.3 and the fact that we are considering only a finite number (in fact, N) $Y\left(t_{k}\right)$ 's. But (B) is true by [2, Theorem 6], and the theorem follows.

THEOREM 4.3. If $X=\{X(t), 0 \leqq t<\infty\}$ is an $L_{1}$ bounded martingale, and if $v$ satisfies the hypotheses of Theorem 4.2, then $\lim _{t \rightarrow \infty} Y(t)$ exists and is finite a.s.

Proof. If the limit exists, then it is finite a.s. by Theorem 4.2. To show that the limit exists, let $U_{a b}$ be the number of upcrossings of the interval $[a, b]$ by the process $Y=\{Y(t), 0 \leqq t<\infty\}$. It will then be sufficient, in the light of well-known arguments, to prove

$$
\lambda P\left\{(b-a) U_{a b}-a^{+}>\lambda\right\} \leqq M \sup _{t} E|X(t)| .
$$

To prove (A), let $t_{1}<t_{2}<\cdots<t_{N}$ be given, and define $U_{a b}\left(t_{1}, \ldots, t_{N}\right)$ to be the number of upcrossings of $[a, b]$ by $Y\left(t_{1}\right), \ldots, Y\left(t_{N}\right)$. We will first prove

$$
\lambda P\left\{(b-a) U_{a b}\left(t_{1}, \ldots, t_{N}\right)-a^{+}>\lambda\right\} \leqq M \sup _{t} E|X(t)| .
$$

To do this, consider step functions $v_{n}$ converging to $v$, and set $Y^{n}(t)=\int_{0}^{t} v_{n}(s) d X(s)$. For each $k, k=1,2, \ldots, N, Y^{n}\left(t_{k}\right) \rightarrow Y\left(t_{k}\right)$ in probability. By a standard theorem and an elementary argument, we can find a subsequence of the $Y^{n}\left(t_{k}\right)$, which we also index by $n$, such that $Y^{n}\left(t_{k}\right) \rightarrow Y\left(t_{k}\right)$ a.s., for each $k=1,2, \ldots, N$. Therefore, if $U_{a b}^{n}\left(t_{1}, \ldots, t_{N}\right)$ denotes the number of upcrossings of $[a, b]$ by $Y^{n}\left(t_{1}\right), \ldots, Y^{n}\left(t_{N}\right)$, then $U_{a b}^{n}\left(t_{1}, \ldots, t_{N}\right)(\omega) \rightarrow U_{a b}\left(t_{1}, \ldots, t_{N}\right)(\omega)$ a.s. Hence, to prove (B), it suffices to prove

$$
\lambda P\left\{(b-a) U_{a b}^{n}\left(t_{1}, \ldots, t_{N}\right)-a^{+}>\lambda\right\} \leqq M \sup _{t} E|X(t)| .
$$

Since this is true by Burkholder's Theorem 7 [2], (B) is also true. The inequality (A) then follows from (B) upon taking the set $\left\{s_{1}, s_{2}, \ldots\right\}$ to be a separant for $Y$, since in this case $U_{a b}\left\{s_{1}, \ldots, s_{N}\right\}(\omega) \rightarrow U_{a b}(\omega)$.

THEOREM 4.4. Let $X=\{X(t), 0 \leqq t<\infty\}$ be a sample continuous martingale, and let $v$ satisfy the hypotheses of Theorem 4.2. Then the process $Y$ is also sample continuous. 
Proof. It suffices to prove the theorem for $t$ restricted to $[0, b]$, where $0<b<\infty$ is arbitrary. The theorem is true if $v$ is a step function. It is also true if $X$ is an $L_{4}$ martingale, and $v$ satisfies the hypotheses of Theorem 4.2. To see this, choose a sequence of bounded step functions $v_{n}$, converging to $v$ in $e-s$ norm, in such a way that $\left\|v-v_{n}\right\|_{e-s} \leqq 1 / n^{2}$. Define $Y^{n}(t)$ by $Y^{n}(t)=\int_{0}^{t} v_{n}(s) d X(s)$. The process $Y^{n}=\left\{Y^{n}(t), t \in[0, b]\right\}$ has continuous paths and the process $Y(t)-Y^{n}(t)$ $=\int_{0}^{t}\left[v(s)-v_{n}(s)\right] d X(s)$ is a martingale. Therefore, by a standard martingale theorem,

$$
\begin{aligned}
P\left\{\sup _{t}\left|Y(t)-Y^{n}(t)\right|>1 / n\right\} & \leqq n^{2} E\left|Y(b)-Y^{n}(b)\right|^{2} \\
& \leqq M_{4} n^{2}\left\|v-v_{n}\right\|_{e-s}^{2} E^{1 / 2}|X(b)|^{4} \leqq \text { constant } / n^{2},
\end{aligned}
$$

using the proof of Lemma 2.2. By Borel-Cantelli, we conclude $\left|Y(t)-Y^{n}(t)\right| \leqq 1 / n$, $0 \leqq t \leqq b$, for all sufficiently large $n$, with probability one. Therefore, with probability one, $Y^{n}(t)$ converges uniformly to $Y(t)$, proving the result for $L_{4}$ martingales, since $Y^{n}(t)$ is continuous for each $n$. To treat the general case, let

$$
T_{n}=\inf \{t:|X(t)|>n\}
$$

(or $T_{n}=b$, if there is no such $t$ ). Let $X^{n}=\left\{X^{n}(t), 0 \leqq t \leqq b\right\}$ be a martingale, with $X^{n}(t)=X\left(T_{n} \wedge t\right)$, and set $Y^{n}(t)=\int_{0}^{t} v(s) d X^{n}(s)$. Since $X^{n}$ has continuous paths, so does $Y^{n}=\left\{Y^{n}(t), 0 \leqq t \leqq b\right\}$, by the result just proved. Moreover, on the $\omega$-set $\left\{\sup _{t}|X(t)|<n\right\}, Y^{n}(t)=Y(t)$, as can be seen by considering step functions and passing to the limit. Therefore, if $\omega \in\left\{\sup _{t}|X(t)|<n\right\}$ the trajectory $Y(\cdot, \omega)$ is continuous (except in a null set). Since $\left\{\sup _{t}|X(t)|<n\right\}$ increases to $\Omega$ as $n$ approaches $\infty$, the proof is complete. The part of the proof concerning $L_{4}$ martingales is modeled on a proof by Doob [4, p. 446], the change being the use of Lemma 2.2 in (1).

5. Stochastic step functions. The completion of the space of $t-\omega$ step functions under the $e-s$ norm provides a reasonably wide and useful class of integrands $v$ for the integrals $\int v(t) d X(t)$. However, it may sometimes be important to know that an even wider class of integrands can be obtained. To effect this generalization we will use the notion of a stochastic step function introduced by Courrège in [3].

We will make the following assumptions which will be assumed to hold throughout this section. We assume that the probability space $(\Omega, F, P)$ is complete with respect to the measure $P$, that each of the sigma fields $F(t)$ contains all the null sets, and that the martingales labeled $X$ have right continuous paths. A nonnegative random variable $\tau$ on $\Omega$ is a stopping time relative to the family $F(t)$ if $\{\tau \leqq t\} \in F(t)$ for each $t$. We will denote by $F(\tau)$ the sigma field

$$
\{A \in F: A \cap\{\tau \leqq t\} \in F(t), 0 \leqq t<\infty\}
$$

The above assumptions imply the fact (convenient below) that if $Z=\{Z(t), 0 \leqq t<\infty\}$ is any process adapted to the $F(t)$ family, and having a.s. right continuous paths (in particular, if $Z=X$ ), then the random variable $Z(\tau)$ is $F(\tau)$ measurable. 
Let $0 \leqq \tau_{1} \leqq \tau_{2} \leqq \cdots, \tau_{n} \uparrow \infty$, be an increasing sequence of stopping times. Then $v=\{v(t), 0 \leqq t<\infty\}$ is an extended stochastic step function if

$$
v(t)=v\left(\tau_{n}\right) \text { on }\left[\tau_{n}, \tau_{n+1}\right), \quad n=1,2, \ldots,
$$

where $v\left(\tau_{n}\right)$ is $F\left(\tau_{n}\right)$ measurable. The stochastic integral of $v$ with respect to the martingale $X$ may then be defined to be

$$
\sum v\left(\tau_{n}\right)\left[X\left(\tau_{n+1}\right)-X\left(\tau_{n}\right)\right]
$$

If the martingale $X$ has a last element $X(\infty)$, then $E\left[X(\infty) \mid F\left(\tau_{n}\right)\right]=X\left(\tau_{n}\right)$, so that in particular, $\sup _{n} E\left|X\left(\tau_{n}\right)\right|<\infty$. It follows from Burkholder's Theorem 1 applied to the martingale $\left\{X\left(\tau_{1}\right), X\left(\tau_{2}\right), \ldots\right\}$ that the expression (1) is well defined and finite on the $\omega$-set $\left\{\sup _{n}\left|v\left(\tau_{n}\right)\right|<\infty\right\}$. If $1<p<\infty$, and if $X$ is further assumed to be $L_{p}$ bounded, then $\left\{X\left(\tau_{1}\right), \ldots\right\}$ is also an $L_{p}$ bounded martingale, so that (1) exists as a limit in $L_{p}$ norm as well, whenever $v$ is uniformly bounded. If each $\tau_{n}$ is taken to be identically constant, the stochastic step function reduces to the ordinary $t-\omega$ step function discussed in $\$ \S 2$ and 3.

Let $v=\{v(t), 0 \leqq t<\infty\}$ satisfy the following conditions:

(2) (b) for every stopping time $\tau, v(\tau)$ is $F(\tau)$ measurable,

(c) $v$ has right continuous paths with left limits.

THEOREM 5.1. If the process $v$ satisfies the conditions (2), then $v$ is in the $e-s$ completion of the (extended) stochastic step functions.

Proof. For $v$ satisfying the given conditions, and for any $\varepsilon>0$, there exists an increasing sequence of stopping times $\tau_{n}$, depending on $\varepsilon$, such that $\tau_{n} \rightarrow \infty$, and

$$
\sup _{s, t \in\left[\tau_{n}, \tau_{n+1}\right)}|v(t)-v(s)|<\varepsilon \quad \text { a.s. }
$$

For each $m, m=1,2, \ldots$, consider the sequence of stopping times $0<\tau_{1}^{m}<\tau_{2}^{m}<\cdots$, $r_{n}^{m} \rightarrow \infty$ as $n \rightarrow \infty$, obtained from (3) by setting $\varepsilon$ equal to $1 / m$. If the stochastic step function $v_{m}$ is defined by $v_{m}(t)=v\left(\tau_{n}^{m}\right)$ on $\left[\tau_{n}^{m}, \tau_{n+1}^{m}\right)$, then $\sup _{t}\left|v_{m}(t)-v(t)\right|<1 / m$ a.s., and so approaches zero as $m \rightarrow \infty$. Since $\left\{h_{m}: h_{m}=\sup _{t}\left|v_{m}(t)-v(t)\right|\right\}$ is uniformly bounded, the dominated convergence theorem applies to show that $E h_{m}^{4} \rightarrow 0$; i.e. $v_{m} \rightarrow v$ in $e-s$ norm.

Next we indicate to what extent the theorems of $\$ 2$ through 4 continue to hold when $t-\omega$ step functions are replaced by the more general stochastic step functions. Few changes will be needed. Recall that if $1<p<\infty$, and if $X$ is an $L_{p}$ bounded martingale, and if $\left\{\tau_{n}\right\}$ is an increasing sequence of stopping times, then $\left\{X\left(\tau_{1}\right), X\left(\tau_{2}\right), \ldots\right\}$ is an $L_{p}$ bounded martingale satisfying $X\left(\tau_{n}\right)=E\left\{X(\infty) \mid F\left(\tau_{n}\right)\right\}$ and $\sup _{n} E\left|X\left(\tau_{n}\right)\right|^{p} \leqq E|X(\infty)|^{p}$. With this observation, the proofs of Theorems 2.3, 2.5, and 4.1 are easily seen to hold in the more general situation, there being only notational adjustments required. If $X$ has a last element, $X(\infty)$, then the martingale 
$\left\{X\left(\tau_{1}\right), X\left(\tau_{2}\right), \ldots\right\} \quad$ is uniformly integrable, $X\left(\tau_{n}\right)=E\left\{X(\infty) \mid F\left(\tau_{n}\right)\right\}$, and $\sup _{n} E\left|X\left(\tau_{n}\right)\right| \leqq E|X(\infty)|$; therefore, we see that Theorem 3.1 also continues to hold. To extend Theorem 4.2, note that, in the light of the preceding remarks, the proof of Theorem 4.2 establishes for each $b, 0<b<\infty$, the inequality

$$
\lambda P\left\{\sup _{0 \leq t \leq b}|Y(t)|>\lambda\right\} \leqq M E|X(b)| \leqq M \sup _{t} E|X(t)| .
$$

The right-hand side is independent of $b$, and $\left\{\sup _{0 \leqq t \leqq b}|Y(t)|>\lambda\right\}$ increases as $b$ increases. Hence, letting $b \rightarrow \infty$, one obtains the desired result. A similar argument establishes Theorem 4.3 in the general case.

Since the argumient of Theorem 4.4 holds in the general case, it remains only to verify that Theorem 3.3 holds. If the martingale $X$ satisfies

$$
\sup _{t} E|X(t)|\left[\log ^{+}|X(t)|\right]^{2},
$$

then the nonnegative submartingale

$$
Y(t)=|X(t)| \log ^{+}|X(t)| \log \log ^{+}|X(t)|
$$

is uniformly integrable. Therefore, from Doob's optional sampling theorem, it follows that if $\tau$ is a stopping time (finite or infinite), then $E Y(\tau) \leqq E Y(\infty)$. Applying the remark after Lemma 3.2, the extended theorem is obtained.

Thus we conclude that the theorems of $\$ \$ 2$ through 4 can be extended virtually without change to the case where $t-\omega$ step functions are replaced by extended stochastic step functions.

The condition (2a) can be weakened considerably. Let $v$ satisfy (2b), (2c), and let $0<c$. Define $v_{c}$ by $v_{c}(t, \omega)=v(t, \omega)$ if $|v(t, \omega)|<c$, and $v_{c}(t, \omega)=c$ otherwise. Then $v_{c}$ satisfies all of the assumptions (2), and so the integral $\int v_{c}(t) d X(t)$ exists. Letting $c \rightarrow \infty$, one sees that, on the set $\left\{\sup _{t}|v(t, \omega)|<\infty\right\}, \int v(t) d X(t)$ exists and is finite. If $\Omega=\left\{\sup _{t}|v(t, \omega)|<\infty\right\}$, then it is seen that, in fact, $\int v(t) d X(t)$ exists as a limit in probability of a sequence of step function integrals.

The equivalent norm property. We conclude this section by constructing a norm $n_{p}^{\prime}$ on the (stochastic) step functions with the property that a sequence of step functions converges in the norm $n_{p}^{\prime}$ if and only if the corresponding step function integrals converge in $L_{p}$ norm. Here we assume $1<p<\infty$.

Let $v=\{v(t), t \in T\}$ be a stochastic step function with $\sup _{t}|v(t)|<\infty$. There may be many increasing sequences of stopping times $\left\{\tau_{n}\right\}$ such that $v$ can be represented in the form

$$
v(t, \omega)=v\left(\tau_{n}, \omega\right) \text { on }\left[\tau_{n}, \tau_{n+1}\right) .
$$

(For example, consider any refinement of an existing sequence of stopping times.) We define a norm of $v$ with respect to the $L_{p}$ bounded martingale $X$ by

$$
n_{p}^{\prime}(v)=\sup \left\{E\left|\sum v\left(\tau_{n}\right)^{2}\left[X\left(\tau_{n+1}\right)-X\left(\tau_{n}\right)\right]^{2}\right|^{p / 2}\right\}^{1 / p}
$$


where here the supremum is taken over all increasing sequences of stopping times with respect to which $v$ has a representation. A manipulation of Burkholder's Theorem 9 yields the following relationship whenever $v$ is a bounded stochastic step function:

$$
\begin{aligned}
& M_{p} E\left|\sum v\left(\tau_{n}\right)^{2}\left[X\left(\tau_{n+1}\right)-X\left(\tau_{n}\right)\right]^{2}\right|^{p / 2} \\
& \quad \leqq E\left|\int v(t) d X(t)\right|^{p} \leqq N_{p} E\left|\sum v\left(\tau_{n}\right)^{2}\left[X\left(\tau_{n+1}\right)-X\left(\tau_{n}\right)\right]^{2}\right|^{p / 2}
\end{aligned}
$$

The middle term does not depend on the particular representation chosen for $v$. Hence, (4) yields

$$
M_{p}^{\prime} n_{p}^{\prime}(v) \leqq\left\|\int v(t) d X(t)\right\|_{p} \leqq N_{p}^{\prime} n_{p}^{\prime}(v) .
$$

The inequality (6) implies that the mapping $v \rightarrow \int v(t) d X(t)$ from the vector space of bounded step functions into a subset of $L_{p}$ satisfies an equivalent norm property: that is, convergence in the space of step functions occurs if and only if there is convergence of the corresponding stochastic integrals in $L_{p}$ norm. In case $p=2$, the mapping $v \rightarrow \int v(t) d X(t)$ is actually an isometry. It follows from (6) and Theorem 2.3 that all bounded processes in the $e-s$ completion of the space of step functions belong to the completion of the step functions under the norm $n_{p}^{\prime}$. The $n_{p}^{\prime}$ completion of the step functions will, by virtue of (6), yield the largest possible class of integrands for which the stochastic integral will exist as an $L_{\mathfrak{p}}$ limit (provided, of course, the integral is originally defined in the way we have adopted). Since the inequality of [2, Theorem 9], is known to fail for $L_{1}$ martingales, a somewhat different theory must be developed for this case (see §8).

In the form given here, the norm $n_{p}^{\prime}$ is difficult to use and it is not readily apparent what processes belong to the completion of the step functions under this norm. In $\$ 8$, a more satisfying formulation is given for martingales $X$ which are right continuous. The reformulation depends on the theory of quadratic variation developed in the next two sections.

6. Quadratic variation. Let $X=\{X(t), F(t), t \in[a, b]\}$ be a martingale, and $\pi=\left\{\pi_{m}, m=1,2, \ldots\right\}$ a sequence of partitions of $[a, b]$ satisfying:

$$
\begin{gathered}
\text { for each } m, \pi_{m} \text { is given by } a \leqq t_{m 1}<t_{m 2}<\cdots<t_{m k_{m}} \leqq b, \\
\max _{k}\left[t_{m, k+1}-t_{m k}\right] \rightarrow 0 \text { as } m \rightarrow \infty .
\end{gathered}
$$

Define $S_{m}(X)$, the quadratic variation of $X$ corresponding to $\pi_{m}$, by

(C) $\left[S_{m}(X)\right]^{2}=\left[X\left(t_{m 1}\right)\right]^{2}+\left[X\left(t_{m 2}\right)-X\left(t_{m 1}\right)\right]^{2}+\cdots+\left[X\left(t_{m k_{m}}\right)-X\left(t_{m, k_{m}-1}\right)\right]^{2}$.

In this section, we first study the behavior of $S_{m}(X)$ as $m \rightarrow \infty$, when $X$ is an a.s. sample continuous martingale. After this, we develop a theory of quadratic variation for arbitrary right continuous martingales. 
Certain cases have already been studied. For example, if it is further supposed that $\pi_{m+1}$ is a refinement of $\pi_{m}$ (which we do not assume in this section), and if we suppose $X$ to be Brownian motion on $[a, b]$, then $\lim _{m \rightarrow \infty} S_{m}(X)$ exists in the sense of a.s. convergence. If $f=\left(f_{1}, f_{2}, \ldots\right)$ is a (discrete) martingale, and $S_{m}^{2}(f)$ $=f_{1}^{2}+\left(f_{2}-f_{1}\right)^{2}+\cdots+\left(f_{m}-f_{m-1}\right)^{2}$ then $\lim _{m \rightarrow \infty} S_{m}(f)$ exists in the sense of a.s. convergence if $f$ is $L_{1}$ bounded (Austin [1]), and in the sense of $L_{p}$ convergence if $f$ is $L_{p}$ bounded, $1<p<\infty$ (Burkholder [2]).

LEMMA 6.1. Let $X=\{X(t), t \in[a, b]\}$ be a uniformly bounded martingale with a.s. continuous paths. Let $\left\{\pi_{m}\right\}$ be a sequence of partitions satisfying (A) and (B). Then,

$$
\lim _{m \rightarrow \infty} \sum X\left(t_{m k}\right)\left[X\left(t_{m, k+1}\right)-X\left(t_{m k}\right)\right]
$$

exists as an $L_{p}$ limit, $1<p<\infty$. The limit is denoted by $\int X(t) d X(t)$.

Proof. Let $X^{m}(t)$ denote the step function corresponding to the partition $\pi_{m}: X^{m}(t)=X\left(t_{m k}\right)$ on $\left[t_{m k}, t_{m, k+1}\right)$. For each $\omega$ (except for a set of probability zero) $X(t, \omega)$ is a continuous function of $t$, and, since the interval is compact, uniformly continuous. Therefore, $\sup _{t}\left|X^{n}(t)-X^{m}(t)\right| \rightarrow 0$ as $m, n \rightarrow \infty$, for the particular $\omega$ chosen. Hence, $\sup _{t}\left|X^{n}(t)-X^{m}(t)\right| \rightarrow 0$ a.s., and since this is uniformly bounded, $E \sup _{t}\left|X^{n}(t)-X^{m}(t)\right|^{4} \rightarrow 0$, by the dominated convergence theorem. Thus, $X^{m}(t)$ is Cauchy in $e-s$ norm, so the integral $\int X(t) d X(t)$ exists as an $L_{p}$ limit, $1<p<\infty$, by Theorem 2.3.

THEOREM 6.2. Let $X=\{X(t), t \in[a, b]\}$ be a martingale with a.s. continuous paths. Then

(a) If $1<p<\infty$ and if $E|X(b)|^{p}<\infty$, then $S_{m}(X) \rightarrow S(X)$ in $L_{p}$ norm.

(b) If $E|X(b)|<\infty$, then $S_{m}(X) \rightarrow S(X)$ in probability.

(c) If $1<p<\infty$, there are positive constants $M_{p}$ and $N_{p}$, depending on $p$ only, such that

$$
M_{p} E S(X)^{p} \leqq E|X(b)|^{p} \leqq N_{p} E S(X)^{p} .
$$

Proof. We remark first that if (a) is true, then (c) is immediate, since the inequality of (c) holds for discrete martingales, by [2, Theorem 9], and so holds in the limit by the convergence in $L_{p}$ of $S_{m}(X)$ to $S(X)$.

We can and will assume for the remainder of the proof that for each partition $\pi_{m}$ we have $t_{m 1}=a, t_{m k_{m}}=b$, since the general case follows readily from this one. Define the stopping time $T_{n}$ by $T_{m}=\inf \{t: a \leqq t \leqq b,|X(t)|>n\} ; T_{n}=b$, if this set is empty. Define the uniformly bounded martingale $X^{n}=\left\{X^{n}(t), t \in[a, b]\right\}$ by $X^{n}(t)$ $=X\left(t \wedge T_{n}\right)$. By a short calculation (see the remark attributed to Doob in [2, p. 1497]), one obtains

$$
S_{m}^{2}\left(X^{n}\right)=\left[X^{n}(b)\right]^{2}-2 \sum X^{n}\left(t_{m k}\right)\left[X^{n}\left(t_{m, k+1}\right)-X^{n}\left(t_{m k}\right)\right]
$$


As $m \rightarrow \infty$, the summation on the right converges in $L_{p}$ norm, by Lemma 6.1 . Therefore, $\lim _{m \rightarrow \infty} S_{m}\left(X^{n}\right)=S\left(X^{n}\right)$ in $L_{p}$ norm. By the definition of $T_{n}$

$$
S_{m}\left(X^{n}\right)=S\left(X^{k}\right)=S_{m}(X)
$$

on the set $\left\{T_{k}=\infty\right\}=\left\{\sup _{t}|X(t)| \leqq k\right\}$, assuming $k \leqq n$. Hence, $S_{m}\left(X^{n}\right)$ converges to $S_{m}(X)$ in probability, uniformly in $m$, since

Writing

$$
P\left\{\left|S_{m}(X)-S_{m}\left(X^{j}\right)\right|>\delta\right\} \leqq P\{\sup |X(t)|>j\} \leqq \int|X(b)| \mid j .
$$

$$
\begin{aligned}
P\left\{\left|S_{m}(X)-S_{n}(X)\right|>\varepsilon\right\} \leqq & P\left\{\left|S_{m}(X)-S_{m}\left(X^{j}\right)\right|>\varepsilon / 3\right\}+P\left\{\left|S_{m}\left(X^{j}\right)-S_{n}\left(X^{j}\right)\right|>\varepsilon / 3\right\} \\
& +P\left\{\left|S_{n}\left(X^{j}\right)-S_{n}(X)\right|>\varepsilon / 3\right\},
\end{aligned}
$$

one now sees that $S_{m}(X)$ converges in probability to a random variable $S(X)$, proving (b).

To prove the statement (a) concerning $L_{p}$ convergence, $1<p<\infty$, it will suffice to prove that

$$
E\left|S_{m}\left(X^{n}\right)-S_{m}(X)\right|^{p} \rightarrow 0 \quad \text { as } n \rightarrow \infty
$$

uniformly in $m$, since we have already shown that $\lim _{m \rightarrow \infty} S_{m}\left(X^{n}\right)=S\left(X^{n}\right)$ in $L_{p}$ norm. We have

$$
E\left|S_{m}\left(X^{n}\right)-S_{m}(X)\right|^{p} \leqq E\left|S_{m}^{2}\left(X^{n}\right)-S_{m}^{2}(X)\right|^{p / 2} .
$$

Let $A_{j}=X^{n}\left(t_{m, j+1}\right)-X^{n}\left(t_{m j}\right)$ and $B_{j}=X\left(t_{m, j+1}\right)-X\left(t_{m j}\right)$. Then, continuing as before,

$$
\begin{aligned}
E\left|S_{m}\left(X^{n}\right)-S_{m}(X)\right|^{p} \leqq & E\left|\sum\left(A_{k}^{2}-B_{k}^{2}\right)\right|^{p / 2} \\
\leqq & K_{p} E\left|\sum A_{k}\left(A_{k}-B_{k}\right)\right|^{p / 2} \\
& +K_{p} E\left|\sum B_{k}\left(A_{k}-B_{k}\right)\right|^{p / 2}
\end{aligned}
$$

where $K_{p}$ is a positive constant depending on $p$ only. The first term on the right does not exceed

$$
\begin{aligned}
K_{p} E \sum\left|A_{k}\right|\left|A_{k}-B_{k}\right|^{p / 2} & \leqq K_{p} E\left|\left[\sum A_{k}^{2}\right]^{1 / 2}\left[\sum\left(A_{k}-B_{k}\right)^{2}\right]^{1 / 2}\right|^{p / 2} \\
& \leqq K_{p} E\left[\sum A_{k}^{2}\right]^{p / 4}\left[\sum\left(A_{k}-B_{k}\right)^{2}\right]^{p / 4} \\
& \leqq K_{p} E^{1 / 2}\left[\sum A_{k}^{2}\right]^{p / 2} E^{1 / 2}\left[\sum\left(A_{k}-B_{k}\right)^{2}\right]^{p / 2} \\
& \leqq K_{p} E^{1 / 2} S_{m}\left(X^{n}\right)^{p} E^{1 / 2} S_{m}\left(X^{n}-X\right)^{p} \\
& \leqq\left(K_{p} \mid M_{p}\right) E^{1 / 2}|X(b)|^{p} E^{1 / 2}\left|X^{n}(b)-X(b)\right|^{p}
\end{aligned}
$$

using [2, Theorem 9]. Since the last expression approaches zero, uniformly in $m$, and since a similar result holds for the second term on the right, the proof is complete.

THEOREM 6.3. Let $X=\{X(t), t \in[a, b]\}$ be a martingale with almost all paths continuous and of bounded variation. Then $P\{X(0) \equiv X(t), t \in[a, b]\}=1$. 
This theorem has been obtained by D. Fisk [5], using a different proof.

Proof. We may assume that $X(0) \equiv 0$, for, if not, we just consider the martingale $X(t)-X(0)$. We prove the theorem first for the case in which $X$ is a square integrable martingale.

We suppose that if $\Lambda=\{\omega: X(t) \not \equiv 0, t \in[a, b]\}$, then $P(\Lambda)>0$, and derive a contradiction. Since, for each $\omega, X(t, \omega)$ is a continuous function of $t$, it follows that if $\omega \in \Lambda$, then $\int_{0}^{b}|X(t, \omega)| d t>0$; so that $\int_{\Lambda} \int_{0}^{b}|X(t, \omega)| d t d P>0$. Applying Fubini, we have $0<\int_{0}^{b} \int_{\Lambda}|X(t, \omega)| d P d t$, implying that for at least one $t=t_{0}$, $\int_{\Lambda}\left|X\left(t_{0}, \omega\right)\right| d P>0$. Hence $E\left|X\left(t_{0}, \omega\right)\right|>0$, and since $X$ is a submartingale, $0<E\left|X\left(t_{0}\right)\right| \leqq E|X(b)|$. By Theorem 6.2(c), $0<E|X(b)|^{p} \leqq N_{p} E S(X)^{p}$, which implies that $S(X)$ is strictly positive on a set of positive probability. If $\pi_{m}$ is a sequence of partitions satisfying (A) and (B) of this section, then $S_{m}(X) \rightarrow S(X)$ in $L_{p}$ norm, so that there is a subsequence of this sequence of partitions (which we label as before) such that $S_{m}^{2}(X) \rightarrow S^{2}(X)$ a.s. Hence, for all sufficiently large $m$,

$$
\sum\left[X\left(t_{m, k+1}\right)-X\left(t_{m k}\right)\right]^{2} \geqq \delta>0
$$

on a set of positive probability. Therefore,

$$
\delta /\left[\max _{k}\left|X\left(t_{m, k+1}\right)-X\left(t_{m k}\right)\right|\right] \leqq \sum\left|X\left(t_{m, k+1}\right)-X\left(t_{m k}\right)\right|
$$

on a set of positive probability. By the continuity of paths $\max _{k}\left|X\left(t_{m, k+1}\right)-X\left(t_{m k}\right)\right|$ $\rightarrow 0$ as $m \rightarrow \infty$ implying that for $\omega$ in a set of positive probability, the paths are not of bounded variation. This proves the theorem for $L_{p}$ bounded martingales.

To prove the general case, define the stopping time $T_{n}$ by

$$
T_{n}=\inf \{t: 0 \leqq t \leqq b,|X(t)|>n\},
$$

$T_{n}=b$ if the given set is empty. Let $X^{n}(t)$ be the martingale $X\left(t \wedge T_{n}\right)$. Then $X^{n}$ is a square integrable martingale, $X^{n}(0) \equiv 0$. By the result just proved, the path $X^{n}(\cdot, \omega)$ is constant, and equal to zero for almost all $\omega$. The paths $X^{n}(\cdot, \omega)$ are the same as $X(\cdot, \omega)$ when $\omega \in\left\{\omega: \sup _{t}|X(t)|<n\right\}=\Omega_{n}$. Since $\Omega_{n} \uparrow \Omega$, almost all of the paths of $X$ must also be constant.

7. Quadratic variation in right continuous martingales. The purpose of this section is to present some results on quadratic variation for martingales whose paths are not necessarily a.s. continuous. Let $X=\{X(t), t \in[0, b]\}$ be a martingale having a.s. right continuous paths. For integers $m=1,2, \ldots$, let $\varepsilon_{m}>0, \varepsilon_{m} \rightarrow 0$ as $m \rightarrow \infty$. Since $X$ is a process with right continuous paths having left limits, there exists for each $m$ an increasing sequence of stopping times $\tau_{m 1} \leqq \tau_{m 2} \leqq \tau_{m 3} \leqq \cdots$, satisfying

(a) $\tau_{m 1}=0, \tau_{m n} \uparrow b$ as $n \rightarrow \infty$, for each $m$,

(b) $\sup _{t, s \in\left[\tau_{m n}, \tau_{m, n+1}\right)}|X(t)-X(s)| \leqq \varepsilon_{m}$.

If desired, it may also be assumed that $\sup _{k}\left|\tau_{m, k+1}-\tau_{m k}\right| \rightarrow 0$ as $m$ increases, and 
even that $\left\{\tau_{m+1,1}, \tau_{m+1,2}, \tau_{m+1,3}, \ldots\right\}$ is a refinement of $\left\{\tau_{m 1}, \tau_{m 2}, \ldots\right\}$. However, we do not make these assumptions. Define $S\left(X, \tau_{m}\right)$ by

$$
S^{2}\left(X, \tau_{m}\right)=\left[X\left(\tau_{m 1}\right)\right]^{2}+\left[X\left(\tau_{m 2}\right)-X\left(\tau_{m 1}\right)\right]^{2}+\cdots
$$

$S\left(X, \tau_{m}\right)$, the nonnegative square root is finite a.s. for each $m$ by Austin's theorem [1], and Doob's optional sampling theorem.

THeOREM 7.1. Let $X=\{X(t), t \in[a, b]\}$ be a martingale with right continuous paths, and $\left\{\tau_{m k}: m=1,2, \ldots, k=1,2, \ldots\right\}$ a family of stopping times satisfying (a) and (b) for some sequence $\left\{\varepsilon_{m}: \varepsilon_{m}>0, \varepsilon_{m} \rightarrow 0\right\}$. Then

(i) $\lim _{m \rightarrow \infty} S\left(X, \tau_{m}\right)=S(X)$ exists as a limit in probability.

(ii) If $1<p<\infty$, and $E|X(t)|^{p}<\infty$, then $\lim _{m \rightarrow \infty} S\left(X, \tau_{m}\right)=S(X)$ exists as an $L_{p^{\prime}}$ limit, $p^{\prime}<p$.

(iii) Under the hypotheses of (ii), there are positive constants $M_{p}^{\prime \prime}$ and $N_{p}^{\prime \prime}$, depending on $p$ only, such that

$$
M_{p}^{\prime \prime} E S(X)^{p} \leqq E|X(b)|^{p} \leqq N_{p}^{\prime \prime} E S(X)^{p} .
$$

The limit $S(X)$ is independent of the collections $\left\{\tau_{m k}\right\}$ and $\left\{\varepsilon_{m}\right\}$ as long as they satisfy (a) and (b).

REMARK. If $X$ is an a.s. sample continuous martingale, then the $S(X)$ obtained by considering stochastic partitions satisfying (a) and (b), and the $S(X)$ obtained by considering the ordinary partitions satisfying (A) and (B) (at the beginning of §6) will be equal a.s. Therefore, the concept of (stochastic) quadratic variation developed here can be considered a generalization of the concept introduced in $\S 6$.

Proof. As before, we have

$$
S^{2}\left(X, \tau_{m}\right)=[X(b)]^{2}-2 \sum X\left(\tau_{m k}\right)\left[X\left(\tau_{m, k+1}\right)-X\left(\tau_{m k}\right)\right] .
$$

However,

$$
\lim _{m \rightarrow \infty} \sum X\left(\tau_{m k}\right)\left[X\left(\tau_{m, k+1}\right)-X\left(\tau_{m k}\right)\right]=\int X(t) d X(t)
$$

exists as a limit in probability. For, define $X^{c}$ by $X^{c}(t)=X(t)$, if $|X(t, \omega)| \leqq c$, and $X^{c}(t)=c$ otherwise. Then $X^{c}$ satisfies the conditions of (2), $\S 5$, and so $X^{c}\left(\tau_{m k}\right)$ $\rightarrow X^{c}(t)$ in $e-s$ norm as $m \rightarrow \infty$, by Theorem 5.1. Hence, by the extended version of Theorem 2.3 or 3.1 ,

$$
\sum X^{c}\left(\tau_{m k}\right)\left[X\left(\tau_{m, k+1}\right)-X\left(\tau_{m k}\right)\right] \rightarrow \int X^{c}(t) d X(t) .
$$

Moreover,

$$
\begin{aligned}
P & \left\{S^{2}\left(X, \tau_{m}\right)-S^{2}\left(X, \tau_{n}\right) \mid>\gamma\right\} \\
& \leqq P\left\{\left|\sum X^{c}\left(\tau_{m k}\right)\left[X\left(\tau_{m, k+1}\right)-X\left(\tau_{m k}\right)\right]-\sum X\left(\tau_{m k}\right)\left[X\left(\tau_{m, k+1}\right)-X\left(\tau_{m k}\right)\right]\right|>\gamma / 3\right\} \\
& +P\left\{\left|\sum X^{c}\left(\tau_{m k}\right)\left[X\left(\tau_{m, k+1}\right)-X\left(\tau_{m k}\right)\right]-\sum X^{c}\left(\tau_{n k}\right)\left[X\left(\tau_{n, k+1}\right)-X\left(\tau_{n k}\right)\right]\right|>\gamma / 3\right\} \\
& +P\left\{\left|\sum X^{c}\left(\tau_{n k}\right)\left[X\left(\tau_{n, k+1}\right)-X\left(\tau_{n k}\right)\right]-\sum X\left(\tau_{n k}\right)\left[X\left(\tau_{n, k+1}\right)-X\left(\tau_{n k}\right)\right]\right|>\gamma / 3\right\} .
\end{aligned}
$$


From the definition of $X^{c}$,

$$
\begin{aligned}
& P\left\{\left|\sum X^{c}\left(\tau_{m k}\right)\left[X\left(\tau_{m, k+1}\right)-X\left(\tau_{m k}\right)\right]-\sum X\left(\tau_{n k}\right)\left[X\left(\tau_{n, k+1}\right)-X\left(\tau_{n k}\right)\right]\right|>\gamma / 3\right\} \\
& \leqq P\left\{\sup _{t}|X(t)|>c\right\} \leqq(1 / c)^{p} E|X(t)|^{p} .
\end{aligned}
$$

Choose $c$ such that the first and third terms on the right are each less than $\varepsilon / 3$; and then choose $m, n$ so that the middle term is less than $\varepsilon / 3$. This establishes equation (2) and hence (i). Note that if $X$ is a uniformly integrable martingale on $[0, \infty)$, then we can obtain $S\left(X, \tau_{m}\right) \rightarrow S(X)$ for the interval $[0, \infty)$ instead of $[0, b]$.

If it is supposed that $1<p<\infty$, then, since $M_{p} E S\left(X, \tau_{m}\right)^{p} \leqq E|X(b)|^{p}$, it follows that $S\left(X, \tau_{m}\right) \rightarrow S(X)$ in $L_{p^{\prime}}$ norm, whenever $p^{\prime}$ satisfies $1<p^{\prime}<p$, and for such $p^{\prime}$, [2, Theorem 9] yields

$$
M_{p^{\prime}} E S(X)^{p^{\prime}} \leqq E|X(b)|^{p^{\prime}} \leqq N_{p^{\prime}} E S(X)^{p^{\prime}} .
$$

By a straightforward argument using monotone and dominated convergence, one obtains, letting $p^{\prime} \uparrow p$,

$$
M_{p}^{n} E S(X)^{p} \leqq E|X(b)|^{p} \leqq N_{p}^{n} E S(X)^{p}
$$

where $M_{p}^{\prime \prime}, N_{p}^{\prime \prime}$ are positive constants depending only on $p$.

8. Integrals for right continuous martingales. The stochastic integral constructed in $\$ \$ 2,3$, and 5 , using the $e-s$ norm, is perhaps limited in certain respects. It was convenient to consider such an integral in order to obtain the quadratic variation theory of $\$ 7$. Now that this theory has been developed we are in a position to redefine the integral in a more satisfactory manner. In the following we shall use the phrase "right continuous martingale" as an abbreviation for "martingale with a.s. right continuous paths."

If $X=\{X(t), 0 \leqq t<\infty\}$ is a right continuous martingale, the theory of $\$ 7$ implies that for each $t, 0 \leqq t<\infty$, we may obtain

$$
S^{2}(X)_{t}=X^{2}(t)-2 \int_{0}^{t} X(s) d X(s),
$$

which we will denote by $S^{2}(t)$. The process $S^{2}=\left\{S^{2}(t), 0 \leqq t<\infty\right\}$ thus obtained is an increasing process with a.s. right continuous paths. A process $v=\{v(t), 0 \leqq t<\infty\}$ is a left continuous step function if there is an increasing sequence of real numbers $0 \leqq a_{1}<\cdots<a_{n}<\infty$, such that

$$
\begin{aligned}
v(t) & =0, & & t \leqq a_{1}, \\
& =v(j), & & a_{j}<t \leqq a_{j+1}, \\
& =0, & & a_{n}<t,
\end{aligned}
$$

where $v(j)$ is measurable with respect to $F\left(a_{j}\right)$. The integral of $v$ with respect to the martingale $X$ is then defined by equation (1) of $\$ 2$. The reason for adopting left 
continuous step functions in the present section will appear shortly; right continuous step functions were, of course, particularly convenient in proving Theorem 7.1 .

If $v$ is a left continuous step function (or, for that matter, any process integrable $d S^{2}$ ), and if $X$ is a right continuous martingale in $L_{p}$ for some $p$ satisfying $1<p<\infty$, then we define the norm $n_{p}$ by

$$
n_{p}(v)=E\left|\int v^{2}(t) d S^{2}(t)\right|^{p / 2}
$$

where, for each $\omega, \int v^{2}(t) d S^{2}(t)$ is the Lebesgue-Stieltjes integral. If $v$ is given by (1), then it follows from the right continuity of $S^{2}(t)$ and the left continuity of $v$ that, for each $\omega$,

$$
\int v^{2}(t) d S^{2}(t)=\sum_{j=1}^{n} v^{2}(j)\left[S^{2}\left(a_{j+1}\right)-S^{2}\left(a_{j}\right)\right] .
$$

Using Theorem 7.1 and formula (3) above, together with such considerations as appeared in the last part of $\S 5$ (especially the fact that $\int v(t) d X(t)$ does not depend on the step function representation of $v$ ), one obtains for bounded step functions $v$ :

$$
M_{p} n_{p}(v) \leqq E\left|\int v(t) d X(t)\right|^{p} \leqq N_{p} n_{p}(v)
$$

where $M_{p}$ and $N_{p}$ are positive constants depending on $p$ only. Note that if we had not considered left continuous step functions, then (3) would not hold as stated, and thus (4) would not follow. The inequality (4) implies that a sequence of step functions converges in the norm $n_{p}$ if and only if there is convergence in $L_{p}$ norm of the corresponding step function integrals. Thus the definition of the stochastic integral is extended to the completion of the space of bounded left continuous step functions under the norm $n_{p}$. It is easy to see that an analogue of Theorem 4.1 holds in the present case.

In the case $X$ is only a right continuous martingale in $L_{1}$, the preceding theory must be modified. One obtains $S^{2}(t)$ as before; and if it is known that $S(t)$ exists as an $L_{1}$ limit for each $t$, one defines the norm of a bounded left continuous step function $v$ by

$$
n_{1}(v)=E\left|\int v^{2}(t) d S^{2}(t)\right|^{1 / 2}
$$

Using Theorem 8 of [2], one may then obtain

$$
\lambda P\left\{\left|\int v(t) d X(t)\right|>\lambda\right\} \leqq M n_{1}(v)
$$

where $M$ is a positive constant not depending on $v, X$, or $\lambda$. Thus, if $v_{n}$ is a sequence of step functions converging in the norm $n_{1}$, then $\int v_{n}(t) d X(t)$ converges in probability. (As a partial converse [2, Theorem 8 ], also implies that if $v_{n}$ is a sequence 
of step functions such that $\int v_{n}(t) d X(t)$ converges in $L_{1}$ norm, then $\int v_{n}^{2}(t) d S^{2}(t)$ converges in probability.) A simple hypothesis guaranteeing that $S(t)$ exists as an $L_{1}$ limit for each $t$ is that $X$ be $L \log ^{2} L$ bounded, as may be verified using an argument similar to those of Lemma 3.2 and [2, Theorem 10]. It is easily verified that Theorems 4.2 and 4.3 continue to hold whenever $v$ satisfies $|v| \leqq 1$ and belongs to the $n_{1}$ completion of the space of left continuous step functions.

ACKNOWLEDGMENT. The author expresses his sincere appreciation to Professor D. L. Burkholder for helpful advice and encouragement during the preparation of this paper.

\section{REFERENCES}

1. D. G. Austin, A sample function property of martingales, Ann. Math. Statist. 37 (1966), 1396-1397.

2. D. L. Burkholder, Martingale transforms, Ann. Math. Statist. 37 (1966), 1494-1505.

3. P. Courrège, Intégrales stochastiques et martingales de carré intégrable, Seminaire de théorie du potential, Institut Henri Poincaré, Secrétariat mathematique, Paris, 7ième année, 1962, 1963.

4. J. L. Doob, Stochastic processes, Wiley, New York, 1953.

5. D. L. Fisk, Quasi-martingales, Trans. Amer. Math. Soc. 120 (1965), 369-389.

6. P. A. Meyer, A decomposition theorem for supermartingales, Illinois J. Math. 6 (1962), 193-205.

7. — Probability and potentials, Blaisdell, Waltham, Mass., 1966.

8. _ Intégrales stochastiques. II, Seminaire de Probabilités I, Université de Strasbourg, 1967.

9. A. Zygmund, Trigonometric series. I, II, Cambridge Univ. Press, New York, 1959.

UNIVERSITY OF ILLINOIS,

URBANA, IllinoIS 